PROCEEDINGS OF THE

AMERICAN MATHEMATICAL SOCIETY

Volume 133, Number 1, Pages 163-165

S 0002-9939(04)07600-2

Article electronically published on July 26, 2004

\title{
A B.A.I. PROOF OF THE NON-ARENS REGULARITY OF $L^{1}(\mathcal{G})$
}

\author{
COLIN C. GRAHAM
}

(Communicated by Andreas Seeger)

\begin{abstract}
The non-Arens regularity of $L^{1}(\mathcal{G})$ is proved for all non-discrete groups and all amenable groups. The proof uses bounded approximate identities in an elementary way.
\end{abstract}

\section{INTRODUCTION}

A Banach algebra is Arens regular if the two multiplications $\square, \diamond$ of Arens [1] agree on $\mathcal{A}^{* *}$. The $L^{1}$-algebra of a locally compact group is not Arens regular, as proved in [2] for abelian groups and amenable discrete groups, and [10] for the general case. See [5] and [4] for surveys.

Theorem. Let $\mathcal{G}$ be a locally compact group. If $\mathcal{G}$ is either non-discrete or amenable, then $L^{1}(\mathcal{G})$ is not Arens regular and the radical of $L^{1}(\mathcal{G})^{* *}$ has dimension at least $c$.

Remarks. (i). The result is a slight improvement on 2]. The proof here is simpler than [2], the reason for this note. An advantage of this proof is that it constructs an orthogonal (almost orthogonal in the discrete case) sequence, even for nonmetrizable $\mathcal{G}$. For a quick non-constructive proof using Rosenthal's theorem, see 7] or [6].

(ii). This proof was suggested by the idea of Day points in Granirer [7. Day points use a generalized version of the notion of a bounded approximate identity containing more-or-less mutually singular elements to conclude much more than non-Arens regularity. We do not know how to apply Day points in the nonamenable, discrete case. For results on Day points, see [7, 6].

(iii). Ülger [9] shows that a weakly sequentially complete Banach algebra with a b.a.i. is not Arens regular.

\section{The Arens multiplications}

The multiplication $\square$ on the second dual of the Banach algebra $\mathcal{A}$ is $x \square y=$ $\lim _{\alpha} \lim _{\beta} x_{\alpha} y_{\beta}$. The second Arens multiplication $\diamond$ is defined identically, except that the order of the limits is swapped. Here, $x_{\alpha}, y_{\beta} \in \mathcal{A}$ are bounded nets converging to $x, y \in \mathcal{A}^{* *}$, respectively. See [4, (1.5)] and [3, p. $\left.249 \mathrm{ff}\right]$ and their references.

Received by the editors September 10, 2003.

2000 Mathematics Subject Classification. Primary 43A10, 46H99, 46B10.

Key words and phrases. Arens regularity, bounded approximate identity, Day point, locally compact group. 
Proof of Theorem: non-discrete case. We find a sequence $W_{n}$ of neighbourhoods of the identity of $\mathcal{G}$. Let $\mathcal{U}=\left\{U_{\alpha}\right\}$ be a neighbourhood base at the identity of $G$ consisting of relatively compact open sets. Then $m\left(U_{\alpha}\right)^{-1} \chi_{U_{\alpha}}$ is a bounded approximate identity for $L^{1}(G)$. Here $m$ denotes Haar measure.

Let $W_{1}, W_{1}^{\prime} \in \mathcal{U}$ be such that $W_{1} \supset\left(\overline{W_{1}^{\prime} W_{1}^{\prime}}\right)$, but $W_{1} \neq \overline{W_{1}^{\prime} W_{1}^{\prime}}$. Assume $n \geq 2$ and that $W_{j}, W_{j}^{\prime}, 1 \leq j \leq n$, have been found such that for $V_{j}=W_{j} \backslash \overline{W_{j}^{\prime} W_{j}^{\prime}}$, $f_{j}=m\left(V_{j}\right)^{-1} \chi_{V_{j}}$, and $1 \leq j \leq n$ :

$$
\begin{aligned}
& W_{j} \supset \overline{W_{j}^{\prime} W_{j}^{\prime}}, \quad W_{j} \neq \overline{W_{j}^{\prime} W_{j}^{\prime}}, \text { and } \\
& \left\|f_{j}-f_{j} * f_{k}\right\|<2^{-j-k+2}, 1 \leq j<k \leq n .
\end{aligned}
$$

We now find a $U_{\alpha} \in \mathcal{U}$ such that $\overline{U_{\alpha} U_{\alpha}}$ is a proper subset of $W_{n}$. Then there exists a $\gamma>\beta \geq \alpha$ such that for $W_{n+1}=U_{\beta}$ and $W_{n+1}^{\prime}=U_{\gamma}$ the above holds with $n$ replaced by $n+1$.

Now consider the sequence $g_{j}=f_{2 j-1}-f_{2 j}, j \geq 1$. Because the supports of the $f_{j}$ are pairwise disjoint, there exists $S \in L^{\infty}(\mathcal{G})$ such that $\left\langle S, g_{j}\right\rangle=2$ for all $j$ and $\|S\|_{\infty}=1$. Let $G$ be any weak* cluster point of the $g_{j}$ in $L^{1}(G)^{* *}$. Then $\|G\|=2$, since $\|G\| \leq 2 \sup _{j}\left\|f_{j}\right\|_{1}=2$, and $\|G\| \geq\left|\left\langle S, g_{j}\right\rangle\right|=2$.

Let $F$ be any cluster point of the $f_{j}$. Then $\|F\|=1$, by a similar argument. Now, $\lim _{j} \lim _{n} g_{j} * f_{n}=\lim _{j} g_{j}$, because of the limit properties of the $f_{j}$. On the other hand, $\lim _{n} \lim _{j} g_{j} * f_{n}=\lim _{n} f_{n}-f_{n}=0$, because of the limit properties of the $f_{j}$. That proves that $G \square F=G$, while $G \diamond F=F \square G=0$. Similarly, $G \square G=0$, so $G$ is in the radical of $L^{1}(\mathcal{G})^{* *}$.

The dimension of the radical is at least $c$. This is because the weak* closures of $\left\{f_{2 j}\right\}$ and $\left\{f_{2 j-1}\right\}$ in $L^{1}(\mathcal{G})^{* *}$ are homeomophic to the Stone-Čech compactification of $\mathbb{N}$, and so each closure has $c$ distinct elements.

Proof of Theorem: discrete amenable case. The proof here uses F $\varnothing$ lner conditions. $A \Delta B$ will denote the symmetric difference of two sets $A, B$. For $n<k<\infty$ let $W_{n}$ be a finite subset of $G$ such that each contains the identity and

$$
\frac{\#\left[\left(W_{k} W_{n}\right) \Delta W_{k}\right]}{\# W_{k}}+\frac{\#\left[\left(W_{n} W_{k}\right) \Delta W_{k}\right]}{\# W_{k}}<10^{-n-k} .
$$

This is possible for discrete amenable $G$, by [8, p. $62 \mathrm{ff}]$. (If $\# W_{n+1}$ is large enough, adding the identity to $W_{n+1}$ won't affect the inequality.) Let $f_{n}=\left(\# W_{n}\right)^{-1} \chi_{W_{n}}$. It then follows that

$$
\lim _{k \rightarrow \infty}\left\|f_{n} * f_{k}-f_{k}\right\|_{1}=0 \text { for all } n .
$$

Indeed, let $n<k<\infty$. Then $f_{n} * f_{k}(x)$ is an average of $\# W_{k}$ terms each of which is either 0 or $1 / \# W_{n}$. This means that

$$
\begin{array}{r}
\left\|f_{n} * f_{k}-f_{k}\right\|_{1}=\sum_{x \in W_{n} \Delta W_{k}} f_{k}(x)+\sum_{x \in W_{k}}\left(f_{k}(x)-f_{n} * f_{k}(x)\right) \\
\leq \#\left(W_{k} \Delta W_{n}\right) / \# W_{k}+\#\left(W_{k} \Delta W_{n}\right) / \# W_{k} \leq 10^{-n-k},
\end{array}
$$

since the mass on $W_{k}$ "removed" by the convolution is exactly the mass on the complement of $W_{k}$. We used equation (0.1) for the last inequality. Similarly, $\lim _{n \rightarrow \infty}\left\|f_{n} * f_{k}-f_{n}\right\|_{1}=0$.

Let $g_{k}=f_{2 k-1}-f_{2 k}, k \geq 1$. By passing to a subsequence of the $f_{j}$ we find $S \in L^{\infty}(G)$ with $\|S\|_{\infty}=1$ and $\lim \left\langle S, g_{j}\right\rangle=2$. 
Let $G$ be any weak* cluster point of the sequence $g_{j}$ in $L^{1}(G)^{* *}$ and $F$ any weak* cluster point of the $f_{j}$. Then

$$
\begin{aligned}
\lim _{n} \lim _{k}\left\langle S, f_{n} * g_{k}\right\rangle & =\lim _{n} \lim _{k}\left\langle S, f_{n} * f_{2 k-1}-f_{n} * f_{2 k}\right\rangle \\
& =\lim _{k}\left\langle S, f_{2 k-1}-f_{2 k}\right\rangle=2,
\end{aligned}
$$

but

$$
\lim _{k} \lim _{n} f_{n} * g_{k}=\lim _{k} \lim _{n} f_{n} * f_{2 k-1}-f_{n} * f_{2 k}=0 .
$$

Hence $F \square G \neq 0$ but $F \diamond G=0$ and $G \square G=0$. The radical has dimension $c$, just as in the non-discrete case.

\section{REFERENCES}

[1] R. Arens, The adjoint of a bilinear operation, Proc. Amer. Math. Soc. 2, 1951, 839-848. MR 0045941 (13:659f)

[2] P. Civin and B. Yood, The second conjugate space of a Banach algebra as an algebra, Pacific J. Math. 11, 1961, 847-870. MR0143056 (26:622)

[3] H. G. Dales, Banach algebras and automatic continuity, Clarendon Press, Oxford, 2000. MR 1816726 (2002e:46001)

[4] and A. T. M. Lau, The second dual of Beurling algebras, Preprint, 2002.

[5] J. Duncan and S. A. R. Hosseiniun, The second dual of a Banach algebra, Proc. Edinburgh Math. Soc., Section A, 84, 1979, 309-325. MR0559675 (81f:46057)

[6] Colin C. Graham, Arens regularity and weak sequential completeness for quotients of the Fourier algebra, Illinois J. Math, 44, 2000, 712-740. MF,1804322 (2001m:43013)

[7] E. E. Granirer, Day points for quotients of the Fourier algebra $A(G)$, extreme nonergodicity of their duals and extreme non-Arens regularity, Illinois J. Math. 40, (No.3), 1996, 402-419. MR, $1407625(98 \mathrm{c}: 43005)$

[8] J.-P. Pier, Amenable Locally Compact Groups, John Wiley \& Sons, New York, 1984. MR:0767264 (86a:43001)

[9] A. Ulger, Arens regularity of weakly sequentially complete Banach algebras, Proc. Amer. Math. Soc. 127, 1999, 3221-3227. MR 1605953(2000b:46080)

[10] N. J. Young, The irregularity of multiplication in group algebras, Quarterly J. Math. (2) 24, 1973, 59-62. MR0320756(47:9290)

Department of Mathematics, University of British Columbia (Mailing address: RR\#1H-46, Bowen Island, British Columbia, Canada V0N 1G0)

E-mail address: ccgraham@alum.mit.edu 